\title{
ROCK ANISOTROPY AND BRITTLENESS FROM LABORATORY ULTRASONIC MEASUREMENTS IN THE SERVICE OF HYDRAULIC FRACTURING
}

\author{
Rafal MOSKA*, Piotr KASZA and Mateusz MASLOWSKI
}

Oil and Gas Institute - National Research Institute, Lubicz 25 A, 31-503 Kraków, Poland

*Corresponding author's e-mail: moska@inig.pl

\begin{tabular}{l} 
ARTICLE INFO \\
\hline Article history: \\
Received 20 November 2017 \\
Accepted 13 March 2018 \\
Available online 20 March 2018 \\
\hline
\end{tabular}

Keywords:

Ultrasonic laboratory measurements

Elastic anisotropy

Brittleness index

\begin{abstract}
Ultrasonic velocity anisotropy in the rock provides information of variability of the dynamic elastic moduli. Young's modulus and Poisson's ratio calculated from waves velocities can be used to determine brittleness index, which is usually used to predict rock susceptibility for hydraulic fracturing.

This paper describes laboratory ultrasonic measurements carried out in order to improve hydraulic fracturing designing. The research was conducted over two types of rock: shale and limestone. The samples were cut out perpendicularly and parallel to the bedding planes. Next they were tested for effective porosity and mineral composition using XRD method. Directionally depended seismic velocities revealed noticeable anisotropy of laminated shale, caused by orientation of the bedding planes and weak anisotropy of limestone. Based on the velocities, dynamic elastic moduli and its anisotropy coefficients were determined. Calculations of brittleness index based on Young's modulus to Poisson's ratio relation and three types of mineral composition brittleness indexes, revealed strong variability in brittleness for both kind of tested formations. These results show, that different types of brittleness indexes should be used complementary, to better describe fracability of the rock.
\end{abstract}

\section{INTRODUCTION}

Hydraulic fracturing is presently a very common technique of well stimulation. It is based on injection of a high pressurized treatment fluid with proppant into a reservoir. High pressure of liquid causes rock breakdown, propagation of inducted fractures, when grains of proppant prevent from close fractures after treatment. Fracture propagation is related, among other things, to the geomechanical properties of the rock. Variability of the dynamic elastic moduli of the rock depends on many factors, such as lithology, mineral composition, porosity, confining and pore pressures (Bourbie et al., 1987; Winkler and Murphy, 1995; Moska, 2017). Rock anisotropy (variation of elastic properties with direction) is an important factor as well. Changes in ultrasonic velocity caused by anisotropy were described by many researchers (e.g. Sarker and Batzle, 2010; Sone and Zoback, 2013; Inks et al., 2015).

In the past, many concepts of rock brittleness definition have been created. They are based on different approaches such as analysis of stress or strain (e.g. Andreev, 1995; Holt et al., 2011), energy balance (e.g. Tarasov and Potvin, 2013), unconfined compressive strength and Brazilian tests (e.g. Gong and Zhao, 2007), mineral composition (e.g. Jarvie et al., 2007; Wang and Gale, 2009), Lame's parameters $\lambda \rho-\mu \rho$ crossplots studies (e.g. Goodway et al., 2010; Perez and Marfurt, 2010) and other.

However, one of the currently most popular brittleness definition is based on relationship between elastic moduli: Young's modulus $(E)$ and Poisson's ratio (v) (Grieser and Bray, 2007; Rickman et al., 2008; Sun et al., 2013; Luan et al., 2014). Rickman et al. (2008) describe, that these components are combined to reflect the rocks ability to fail under stress (Poisson's ratio) and maintain the fracture (Young's Modulus). Ductile shale is not a good reservoir, because the formation will want to heal any natural or hydraulic fractures. But on the other hand, such shales form a good traps preventing from hydrocarbon migration. Brittle shales contain more pre-existing natural fractures, therefore they have the ability to create expanded network of the fractures during stimulation operations. In addition, energy needed to create new fracture in brittle shales is much lower than in ductile shales (Wanniarachchi et al., 2015).

In the case of brittle shales, the conductivity of the fracture is higher so that more hydrocarbons can get through the fractures to the wellbore. In very ductile rocks may arise embedment phenomena, which is pressing of proppant grains into the surface of fracture (Alramahi and Sundberg, 2012). 
Table 1 Macroscopic description of the samples.

\begin{tabular}{|l|l|}
\hline Sample ID & \multicolumn{1}{c|}{ Macroscopic description } \\
\hline $3268-3279$ & Grey shale, fine grained, clearly visible bedding planes \\
\hline $3366-3377$ & Dark gray carbonate rock, fine grained, strong reaction with 5\% $\mathrm{HCl}$ \\
\hline
\end{tabular}

Table 2 Properties of the measured samples.

\begin{tabular}{|c|c|c|c|c|c|c|}
\hline \multicolumn{2}{|l|}{ Sample ID } & $\begin{array}{c}\text { diameter } \varnothing \\
{[\mathrm{mm}]}\end{array}$ & length $l[\mathrm{~mm}]$ & weight $m[\mathrm{~g}]$ & $\begin{array}{c}\text { effective } \\
\text { porosity } p[\%]\end{array}$ & $\begin{array}{c}\text { bulk density } \rho \\
{\left[\mathrm{g} / \mathrm{cm}^{3}\right]}\end{array}$ \\
\hline 3268 Mancos & \multirow{6}{*}{ 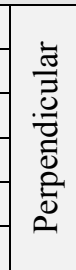 } & 25.39 & 50.95 & 65.00 & 3.73 & 2.525 \\
\hline 3269 Mancos & & 25.39 & 50.95 & 64.09 & 3.74 & 2.532 \\
\hline 3270 Mancos & & 25.39 & 50.95 & 63.78 & 3.36 & 2.530 \\
\hline 3271 Mancos & & 25.39 & 50.95 & 64.24 & 3.45 & 2.537 \\
\hline 3272 Mancos & & 25.39 & 50.95 & 64.11 & 3.57 & 2.563 \\
\hline 3273 Mancos & & 25.39 & 50.95 & 64.04 & 3.19 & 2.536 \\
\hline 3274 Mancos & \multirow{6}{*}{$\begin{array}{l}\overline{\bar{J}} \\
\overline{\tilde{\sigma}} \\
\bar{\sigma}\end{array}$} & 25.39 & 50.95 & 65.52 & 3.52 & 2.525 \\
\hline 3275 Mancos & & 25.39 & 50.95 & 65.64 & 3.42 & 2.529 \\
\hline 3276 Mancos & & 25.39 & 50.95 & 63.75 & 4.20 & 2.543 \\
\hline 3277 Mancos & & 25.39 & 50.95 & 66.17 & 3.74 & 2.543 \\
\hline 3278 Mancos & & 25.39 & 50.95 & 65.70 & 3.50 & 2.528 \\
\hline 3279 Mancos & & 25.39 & 50.95 & 65.18 & 3.25 & 2.529 \\
\hline 3366 Marcellus & \multirow{6}{*}{$\begin{array}{l}\bar{\sigma} \\
\overline{0} \\
\bar{\Xi} \\
\overline{0} \\
\overline{0} \\
0\end{array}$} & 25.55 & 50.50 & 68.63 & 1.27 & 2.646 \\
\hline 3367 Marcellus & & 25.55 & 50.50 & 69.28 & 1.08 & 2.669 \\
\hline 3368 Marcellus & & 25.55 & 50.50 & 69.33 & 0.77 & 2.670 \\
\hline 3369 Marcellus & & 25.55 & 50.30 & 68.83 & 1.22 & 2.668 \\
\hline 3370 Marcellus & & 25.55 & 50.50 & 67.28 & 0.98 & 2.675 \\
\hline 3371 Marcellus & & 25.55 & 50.50 & 68.79 & 1.21 & 2.686 \\
\hline 3372 Marcellus & \multirow{6}{*}{$\begin{array}{l}\bar{\Xi} \\
\stackrel{\bar{\pi}}{\Xi} \\
\tilde{\tilde{a}}\end{array}$} & 25.55 & 50.50 & 69.47 & 0.79 & 2.680 \\
\hline 3373 Marcellus & & 25.55 & 50.50 & 69.31 & 0.98 & 2.674 \\
\hline 3374 Marcellus & & 25.55 & 50.50 & 69.28 & 0.85 & 2.672 \\
\hline 3375 Marcellus & & 25.55 & 50.50 & 68.40 & 0.98 & 2.643 \\
\hline 3376 Marcellus & & 25.55 & 50.50 & 66.76 & 1.17 & 2.581 \\
\hline 3377 Marcellus & & 25.55 & 50.50 & 66.94 & 1.28 & 2.585 \\
\hline
\end{tabular}

Embedment may cause a significant decrease of the fracture conductivity (Britt and Schoeffler, 2009).

Although different types of brittleness indexes are useful many researchers have been questioning its accuracy. Zhang et al. (2016) quote many researchers, who pointed out, that approaches shown by Rickman et al. (2008), Sun et al. (2013) and Luan et al. (2014) assume the same sensitivity of rock brittleness to $E$ and $v$. Determination of rock brittleness using only $E$ and $v$ relations is not accurate, as rock brittleness depends on many other parameters such as bulk modulus and pore pressure. Moreover, Rickman's et al. (2008) approach does not distinguish the brittleness of quartz-rich brittle shales and ductile limestone formations (limestone formations are fracture barriers in the hydrocarbon deposits) (Perez and Marfurt, 2010). In spite of imperfections of $E$ to $v$ relationbased brittleness indexes, they are still used in hydraulic fracturing treatments designing (Zhang et al., 2016).

\section{MATERIALS AND METHODS}

Authors assumed that samples for ultrasonic measurement should be fine-grained, have high content of quartz and clay minerals and relatively low porosity. Therefore it was decided to choose two types of shale rock from North American gas fields: Mancos shale and Marcellus shale. Collected samples originate from the quarries in USA and they were more easily accessible equivalents of rocks from the wellbores. However, during the research it turned out, that Marcellus shale has a significantly high content of calcite, which is not consistent witch data in literature (e.g. McGinley, 2015).

Twelve cylindrical samples from Mancos block and twelve from Marcellus block were cut out. Six samples from each rock were cut out perpendicularly and six along (parallel) to bedding planes. Table 1 presents a preliminary macroscopic description of the samples.

Firstly, an analysis of mineral composition was carried out. Four samples were chosen: two from 


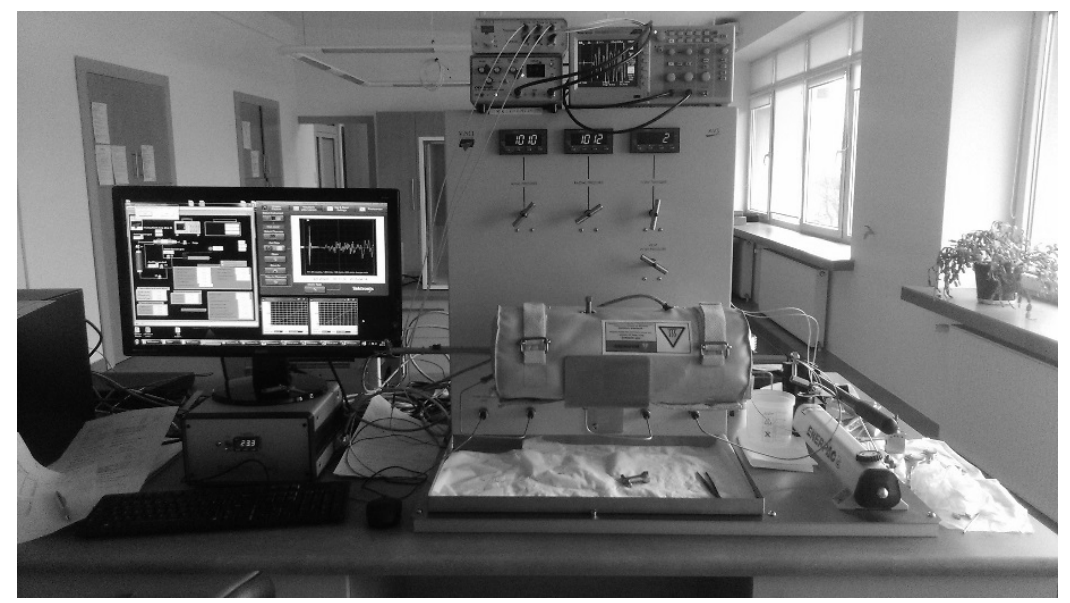

Fig. 1 Ultrasonic apparatus Vinci AVS 700.

Mancos shale and two from Marcellus limestone, cut out perpendicularly and parallel. Analysis were conducted based on x-ray diffraction Rietveld methodology (Środoń et. al., 2001; Kowalska, 2013), using X'Pert apparatus.

Then the dimensions and weights of the samples were determined, with accuracy to $0.01 \mathrm{~mm}$ and $0.01 \mathrm{~g}$ respectively. On this basis, and using HPG-100 helium porosimeter, the effective porosity, and bulk density of the sample were calculated. The results are described in Table 2.

Ultrasonic measurements were conducted using a Vinci AVS 700 apparatus (Fig. 1). The following measurements conditions have established: konventional triaxial stress (confining pressure) 2000 psi $(13.79 \mathrm{MPa})$, room temperature $\left(24-25{ }^{\circ} \mathrm{C}\right)$, dry samples, measurements during first loading, frequency of transducers: $500 \mathrm{MHz}$ (maximum obtained on the transducers). A confining pressure was applied in order to receive higher amplitude of useful signal.

Dynamic elastic moduli were determined using equations which are transformed equations from Fjaer et. al. (2008).

$$
\begin{aligned}
& v=\frac{\frac{1}{2}-\left(\frac{\mathbf{V}_{\mathbf{s}}}{\mathbf{V}_{\mathbf{p}}}\right)^{2}}{1-\left(\frac{\mathbf{V}_{\mathbf{s}}}{\mathbf{V}_{\mathbf{p}}}\right)} \\
& E=\rho \frac{V_{p}^{2}(1+v)(1-v)}{(1-v)}
\end{aligned}
$$

$G=\rho V_{s}^{2}$

$K=\rho V_{p}^{2}-\frac{3}{4} V_{s}^{2}$

where:

$V_{p}-\mathrm{P}$-wave velocity $[\mathrm{m} / \mathrm{s}], V_{s}-\mathrm{S}$-wave velocity $[\mathrm{m} / \mathrm{s}], \rho-$ bulk density $\left[\mathrm{g} / \mathrm{cm}^{3}\right], v-$ Poisson's ratio
[-], E- Young's modulus [Pa], $K$ - bulk modulus [Pa], $G-$ Shear modulus $[\mathrm{Pa}]$.

Next, based on the results of individual core samples, arithmetic averages of parameters were calculated for all core samples cut out in the same direction (Averages in Tables 5-7 and 9-11).

Anisotropy coefficient $k$ was calculated on the basis of the equation presented by Živor et al. (2011) and Stan-Kłeczek (2016):

$k_{V p}=\frac{V_{p \text { max }}-V_{p \min }}{V_{p \text { mean }}} * 100 \%$

where $V_{p \text { mean }}$ is velocity of P-wave, calculated as arithmetical average of velocities measured for all samples cut out perpendicularly and all samples cut out parallel to bedding, and analogously:

$k_{V s}=\frac{V_{s \text { max }}-V_{s \text { min }}}{V_{s \text { mean }}} * 100 \%$

Anisotropy of elastic moduli was calculated using similar equations:

$k_{x}=\frac{x_{\max }-x_{\min }}{x_{\text {mean }}} * 100 \%$

where $x$ is modulus $(v, E, \mathrm{~K}, \mathrm{G}$ or $\lambda)$ and $x_{\text {mean }}$ is value of modulus, calculated as arithmetical average of values of moduli measured in both directions (perpendicularly and parallel).

Brittleness index based on ultrasonic measurements was determined using a method described by Grieser and Bray (2007). The following formulas were used:

$Y M_{\text {BRIT }}=\frac{Y M-Y M_{-} \min }{Y M_{-} \max -Y M_{-} \min } * 100 \%$

$P R_{\text {BRIT }}=\frac{P R-P R_{-} \max }{P R_{-} \text {min }-P R_{-} \max } * 100 \%$ 


$$
B R I T_{\text {Grieser and Bray }, 2007}=\frac{Y M_{-} B R I T+P R_{-} B R I T}{2}
$$

where:

$Y M \_B R I T$ - brittleness from Young's modulus, $P R \_B R I T$ - brittleness from Poisson's ratio, YM measured Young's modulus, $P R$ - measured Poisson's ratio. $Y M \_$min $=0[\mathrm{GPa}], Y M \_\max =100[\mathrm{GPa}]$, $P R \_$min $=0[-], P R \_$max $=0.5[-]$. They are constants defining minimum and maximum values of obtained results. Brittleness index BRIT $T_{\text {Grieser and Bray, } 2007}[\%]$ is in the range between 0 and 100 , where 0 is purely ductile rock and 100 is purely brittle rock.

Brittleness index based on mineral composition was appointed using equations presented below:

- equation from Jarvie et al. (2007),

$$
B R I T_{\text {Jarvieet al., } 2007}=\frac{Q}{Q+D+C+C l}
$$

- modified equation from Wang and Gale (2009):

$$
B R I T_{\text {WangandGale, 2009 }}=\frac{Q+D}{Q+D+C+C l}
$$

This equation (in contrast to Wang's and Gale's equation) does not take into account TOC content. - equation from Jin et al. (2014a, 2014b)

$$
\text { BRIT }_{\text {Jin et al., 2014, 2014b }}=\frac{Q+P l+K f+C+D}{\text { Tot }}
$$

where:

BRIT - brittleness index [-] and symbols corresponds weight percentage quantity of: $Q$ - quartz, $D-$ dolomite, $\mathrm{C}$ - calcite, $\mathrm{Cl}$ - clay minerals, $\mathrm{Pl}-$ plagioclase, $K f$ - potassium feldspar, Tot - weight of total minerals. Mineral brittleness index is in range between 0 and 1 , where 0 is purely ductile rock, and 1 is purely brittle.

\section{RESULTS AND DISCUSSION}

In the Table 3 a mineral composition of the sample, based on the x-ray diffraction measurements are presented.

Table 3 shows that the main construction mineral in Mancos shale is quartz (38.5-40.8 \%). There is a considerable amount of clay minerals as well (34$34.5 \%$ ). Dolomite and other minerals are less significant. Mancos shales examined by Mokhtari (2015) have comparable mineral composition (Table 4). Table 3 reveals also very high calcite content in Marcellus rocks (80-89\%), while quartz content is below $10 \%$. McGinely in his Thesis (2015) quotes data for Marcellus shale samples collected from many locations, which show lower content of calcite, from $0.3 \%$ to $29.0 \%$ (Table 4 ). Increased content of calcite may have noticeable effect on wave velocities, $V p / V s$ ratio and elastic moduli, as Bała (1990) showed.

Tables $5-7$ reveal results of ultrasonic measurements of Mancos shales.

As it shown in Table 5, velocities of P-wave in samples cut out perpendicularly are lower than $\mathrm{P}$-wave velocities in samples cut out parallel. P-wave propagates faster along the boundary between single layers and cracks of the material than perpendicularly to them (Stan-Kłeczek, 2016). Calculated anisotropy coefficient $k$ for P-wave velocity is $11.1 \%$. The variation of the velocity of S-waves are lower $(k=$ $2.6 \%)$. In the research of Sarker and Batzle (2010), on the saturated samples, Mancos B shale exhibits compressional wave anisotropy of about $9 \%$ and shear wave of about $5 \%$. Other researchers present slightly higher values. Table 8 shows anisotropy factors and elastic moduli of Mancos shale available in the literature.

Table 3 Results of quantity analyze of mineral composition on the basis of x-ray diffraction method (XRD). Explanations: percentage by weight: Q - quartz, Pl - plagioclase, K-F - potassium feldspar, C - calcite, $\mathrm{D}$ - dolomite, $\mathrm{An}$ - ankerite, $\mathrm{P}$ - pirite, $\mathrm{M}$ - mica, I - Illite, I/S - mixed-packages illite/smectite, $\mathrm{Ch}$ chlorite, $\mathrm{Kl}$ - kaolinite, $\Sigma \mathrm{Cl}$ - sum of the cay minerále.

\begin{tabular}{|l|c|c|c|c|c|c|c|c|c|c|c|c|c|c|}
\hline \multicolumn{1}{|c|}{ Sample ID } & $\begin{array}{c}Q \\
{[\%]}\end{array}$ & $\begin{array}{c}P l \\
{[\%]}\end{array}$ & $\begin{array}{c}K-F \\
{[\%]}\end{array}$ & $\begin{array}{c}C \\
{[\%]}\end{array}$ & $\begin{array}{c}D \\
{[\%]}\end{array}$ & $\begin{array}{c}A n \\
{[\%]}\end{array}$ & $\begin{array}{c}P \\
{[\%]}\end{array}$ & $\begin{array}{c}M \\
{[\%]}\end{array}$ & $\begin{array}{c}I \\
{[\%]}\end{array}$ & $\begin{array}{c}I / S \\
{[\%]}\end{array}$ & $\begin{array}{c}C h \\
{[\%]}\end{array}$ & $\begin{array}{c}K l \\
{[\%]}\end{array}$ & $\begin{array}{c}\text { Sum } \\
{[\%]}\end{array}$ & $\begin{array}{c}\Sigma C l \\
{[\%]}\end{array}$ \\
\hline 3271 Mancos & 38.5 & 4.0 & 3.8 & 5.6 & 8.0 & 2.6 & 3.0 & 15.4 & 10.0 & 5.2 & 1.6 & 2.3 & 100.0 & 34.5 \\
\hline 3274 Mancos & 40.8 & 3.5 & 4.3 & 4.6 & 7.9 & 2.4 & 2.5 & 22.9 & 6.3 & - & 1.8 & 3.0 & 100.0 & 34.0 \\
\hline 3366 Marcellus & 7.7 & 0.7 & - & 79.7 & 0.9 & 0.7 & 2.2 & 2.7 & 5.4 & - & - & - & 100.0 & 8.1 \\
\hline 3372 Marcellus & 2.1 & - & - & 89.0 & - & - & 5.2 & 1.2 & 2.5 & - & - & - & 100.0 & 3.7 \\
\hline
\end{tabular}

Table 4 Mineral content of Mancos and Marcellus shales by XRD analysis,

${ }^{* 1}$ average from 2 measured samples. ${ }^{* 2}$ depending on the location (5 locations).

\begin{tabular}{|l|c|c|c|c|c|c|}
\hline Mineral & $\begin{array}{c}\text { Mancos, } \\
\text { (Mokhtari, } \\
\text { 2015) }\end{array}$ & $\begin{array}{c}\text { Mancos, } \\
\text { (Sarker and } \\
\text { Batzle, 2010) }\end{array}$ & $\begin{array}{c}\text { Mancos, } \\
(\text { Chandler et } \\
\text { al., 2016) }\end{array}$ & $\begin{array}{c}\text { Mancos, this } \\
\text { study }^{* 1}\end{array}$ & $\begin{array}{c}\text { Marcellus, } \\
\text { (McGinley, }_{2015)^{* 2}}\end{array}$ & $\begin{array}{c}\text { Marcellus, this } \\
\text { study }^{* 1}\end{array}$ \\
\hline Quartz & $43 \%$ & $39 \%$ & $10-25 \%$ & $40 \%$ & $35-67 \%$ & $5 \%$ \\
\hline Carbonates & $22.5 \%$ & $17 \%$ & $6-7 \%$ & $13 \%$ & $0-29 \%$ & $85 \%$ \\
\hline Clay & $20.5 \%$ & $33 \%$ & & $34 \%$ & $11-49 \%$ & $6 \%$ \\
\hline
\end{tabular}


Table 5 Ultrasonic waves velocities and anisotropy of Mancos rock.

\begin{tabular}{|c|c|c|c|c|c|c|c|c|}
\hline \multirow{2}{*}{\multicolumn{2}{|c|}{ Sample ID }} & \multicolumn{2}{|c|}{ Veocity $[\mathrm{m} / \mathrm{s}]$} & \multirow{2}{*}{$\begin{array}{c}\text { Average VP } \\
{[\mathrm{m} / \mathrm{s}]}\end{array}$} & \multirow{2}{*}{$\begin{array}{c}\text { Average VS } \\
{[\mathrm{m} / \mathrm{s}]}\end{array}$} & \multirow{2}{*}{$\begin{array}{l}\text { Average } \\
\text { VP/VS }\end{array}$} & \multirow{2}{*}{$k$ VP [\%] } & \multirow{2}{*}{$k$ VS [\%] } \\
\hline & & P-wave & S-wave & & & & & \\
\hline 3268 Mancos & \multirow{6}{*}{ 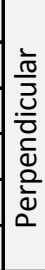 } & & & & - & & & \\
\hline 3269 Mancos & & 3107 & 1716 & \multirow{5}{*}{3082} & \multirow{5}{*}{1656} & \multirow{5}{*}{1.86} & \multirow{11}{*}{11.1} & \multirow{11}{*}{2.6} \\
\hline 3270 Mancos & & 3184 & 1613 & & & & & \\
\hline 3271 Mancos & & 3033 & 1636 & & & & & \\
\hline 3272 Mancos & & 3088 & 1666 & & & & & \\
\hline 3273 Mancos & & 2997 & 1647 & & & & & \\
\hline 3274 Mancos & \multirow{6}{*}{$-\frac{\overline{0}}{\overline{\frac{\pi}{0}}}$} & 3466 & 1702 & \multirow{6}{*}{3444} & \multirow{6}{*}{1700} & \multirow{6}{*}{2.03} & & \\
\hline 3275 Mancos & & 3466 & 1696 & & & & & \\
\hline 3276 Mancos & & 3330 & 1644 & & & & & \\
\hline 3277 Mancos & & 3514 & 1734 & & & & & \\
\hline 3278 Mancos & & 3490 & 1745 & & & & & \\
\hline 3279 Mancos & & 3397 & 1679 & & & & & \\
\hline
\end{tabular}

Table 6 Elastic moduli v, E and anisotropy of Mancos rocks.

\begin{tabular}{|c|c|c|c|c|c|c|c|}
\hline Sample ID & & $v[-]$ & $\begin{array}{c}\text { Average } \\
v[-]\end{array}$ & $k v[\%]$ & $E[\mathrm{GPa}]$ & $\begin{array}{r}\text { Average } \\
E \text { [GPa] }\end{array}$ & $k E[\%]$ \\
\hline 3268 Mancos & \multirow{6}{*}{ 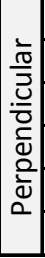 } & & & & & & \\
\hline 3269 Mancos & & 0.28 & \multirow{5}{*}{0.29} & \multirow{12}{*}{14.02} & 19.1 & \multirow{5}{*}{18.0} & \multirow{12}{*}{8.2} \\
\hline 3270 Mancos & & 0.33 & & & 17.5 & & \\
\hline 3271 Mancos & & 0.29 & & & 17.5 & & \\
\hline 3272 Mancos & & 0.29 & & & 18.4 & & \\
\hline 3273 Mancos & & 0.28 & & & 17.6 & & \\
\hline & & & & & & & \\
\hline 3274 Mancos & \multirow{6}{*}{$\frac{\bar{d}}{\overline{\frac{\pi}{\sigma}}}$} & 0.34 & \multirow{6}{*}{0.34} & & 19.6 & \multirow{6}{*}{19.6} & \\
\hline 3275 Mancos & & 0.34 & & & 19.5 & & \\
\hline 3276 Mancos & & 0.34 & & & 18.4 & & \\
\hline 3277 Mancos & & 0.34 & & & 20.4 & & \\
\hline 3278 Mancos & & 0.33 & & & 20.5 & & \\
\hline 3279 Mancos & & 0.34 & & & 19 & & \\
\hline
\end{tabular}

Table 7 Elastic moduli K, G, $\lambda$ and anisotropy of Mancos rocks.

\begin{tabular}{|c|c|c|c|c|c|c|c|c|c|c|}
\hline \multicolumn{2}{|l|}{ Sample ID } & $K[\mathrm{GPa}]$ & $\begin{array}{r}\text { Average } \\
K[\mathrm{GPa}]\end{array}$ & $k K[\%]$ & G [GPa] & $\begin{array}{r}\text { Average } \\
G[\mathrm{GPa}]\end{array}$ & $k \boldsymbol{G}[\%]$ & $\lambda[\mathrm{GPa}]$ & $\begin{array}{c}\text { Average } \\
\lambda[\mathrm{GPa}]\end{array}$ & $k \lambda[\%]$ \\
\hline 3268 Mancos & \multirow{6}{*}{ 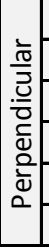 } & \multicolumn{9}{|c|}{ 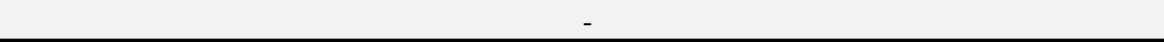 } \\
\hline 3269 Mancos & & 14.5 & \multirow{5}{*}{14.8} & \multirow{12}{*}{30.9} & 7.4 & \multirow{5}{*}{7.0} & \multirow{12}{*}{4.8} & 9.5 & & \multirow{12}{*}{40.6} \\
\hline 3270 Mancos & & 16.9 & & & 6.6 & & & 12.5 & & \\
\hline 3271 Mancos & & 14.2 & & & 6.8 & & & 9.7 & 10.2 & \\
\hline 3272 Mancos & & 14.9 & & & 7.1 & & & 10.2 & & \\
\hline 3273 Mancos & & 13.6 & & & 6.9 & & & 9 & & \\
\hline & & & & & & & & & & \\
\hline 3274 Mancos & \multirow{6}{*}{ 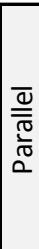 } & 20.5 & \multirow{6}{*}{20.2} & & 7.3 & \multirow{6}{*}{7.3} & & 15.7 & \multirow{6}{*}{15.4} & \\
\hline 3275 Mancos & & 20.6 & & & 7.2 & & & 15.8 & & \\
\hline 3276 Mancos & & 19 & & & 6.9 & & & 14.4 & & \\
\hline 3277 Mancos & & 21.2 & & & 7.6 & & & 16.1 & & \\
\hline 3278 Mancos & & 20.5 & & & 7.7 & & & 15.3 & & \\
\hline 3279 Mancos & & 19.6 & & & 7.1 & & & 14.9 & & \\
\hline
\end{tabular}


Table 8 Ultrasonic properties of Mancos shale. ${ }^{* 1}$ stress-strain test with pulse transmission, saturated samples; ${ }^{* 2}$ based on methodology of Thomsen $(1986) ;{ }^{* 4}$ ultrasonic measurements, dry samples; ${ }^{* 4}$ average from all measured samples.

\begin{tabular}{|c|c|c|c|c|}
\hline Parameter & $\begin{array}{c}\text { Sarker and } \\
\text { Batzle, 2010 }\end{array}$ & $\begin{array}{c}\text { Chandler et al., 2016 after } \\
\text { Thomsen, 1986; } \\
\text { Berryman, 2008 }\end{array}$ & $\begin{array}{c}\text { Chandler et al., } \\
2016^{* 3}\end{array}$ & This study \\
\hline P-wave anisotropy & $9 \%$ & $27 \%$ & $22 \%$ & $11.1 \%$ \\
\hline S-wave anisotropy & $5 \%$ & $13 \%$ & $11 \%$ & $2.6 \%$ \\
\hline Young's Modulus & - & - & $24.8 \mathrm{GPa}$ & $18.8 \mathrm{GPa}^{{ }^{*} 4}$ \\
\hline Poisson's Ratio & - & - & $0.08-0.23$ & $0.31^{* 4}$ \\
\hline
\end{tabular}

Table 9 Ultrasonic waves velocities of Marcellus rock.

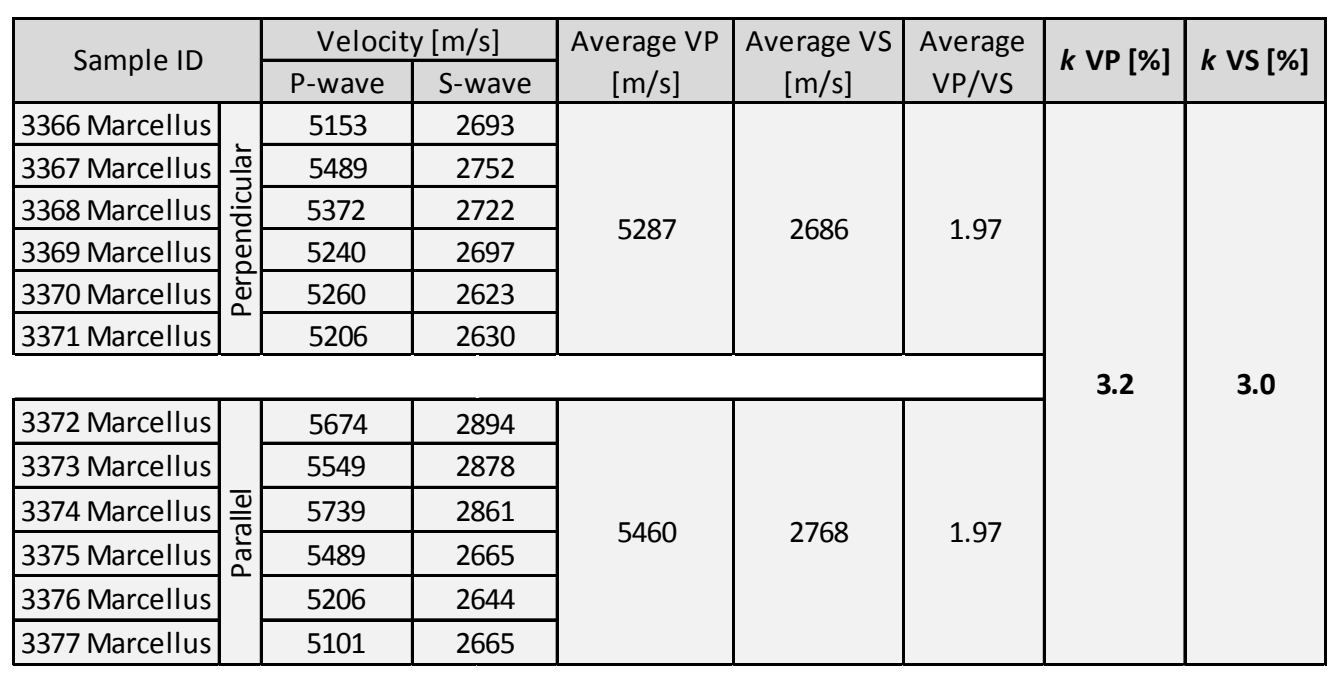

Table 10 Elastic moduli v, E and anisotropy of Marcellus rocks.

\begin{tabular}{|c|c|c|c|c|c|c|c|}
\hline Sample ID & & $v[-]$ & $\begin{array}{c}\text { Average } \\
v[-]\end{array}$ & $k v[\%]$ & $E[\mathrm{GPa}]$ & $\begin{array}{r}\text { Average } \\
E[\mathrm{GPa}]\end{array}$ & $k E[\%]$ \\
\hline 3366 Marcellus & & 0.31 & \multirow{6}{*}{0.33} & \multirow{13}{*}{0.0} & 50.4 & \multirow{6}{*}{51.1} & \multirow{13}{*}{5.1} \\
\hline 3367 Marcellus & $\frac{\pi}{2}$ & 0.33 & & & 53.9 & & \\
\hline 3368 Marcellus & $\frac{. \bar{v}}{0}$ & 0.33 & & & 52.5 & & \\
\hline 3369 Marcellus & 灾 & 0.32 & & & 51.2 & & \\
\hline 3370 Marcellus & $\overline{0}$ & 0.33 & & & 49.0 & & \\
\hline 3371 Marcellus & & 0.33 & & & 49.3 & & \\
\hline & & & & & & & \\
\hline 3372 Marcellus & \multirow{6}{*}{$\mid \begin{array}{c}\overline{\frac{\sigma}{\sigma}} \\
\frac{\bar{\sigma}}{\sigma}\end{array}$} & 0.32 & \multirow{6}{*}{0.33} & & 59.4 & \multirow{6}{*}{53.7} & \\
\hline 3373 Marcellus & & 0.32 & & & 58.2 & & \\
\hline 3374 Marcellus & & 0.33 & & & 58.3 & & \\
\hline 3375 Marcellus & & 0.35 & & & 50.5 & & \\
\hline 3376 Marcellus & & 0.33 & & & 47.8 & & \\
\hline 3377 Marcellus & & 0.31 & & & 48.1 & & \\
\hline
\end{tabular}


Table 11 Elastic moduli K, G, $\lambda$ and anisotropy of Marcellus rocks.

\begin{tabular}{|c|c|c|c|c|c|c|c|c|c|c|}
\hline Sample ID & & $K[\mathrm{GPa}]$ & \begin{tabular}{|c|} 
Average \\
$K$ [GPa]
\end{tabular} & $k K$ [\%] & $G$ [GPa] & $\begin{array}{c}\text { Average } \\
G \text { [GPa] }\end{array}$ & $k G$ [\%] & $\lambda[\mathrm{GPa}]$ & $\begin{array}{c}\text { Average } \\
\lambda \text { [GPa] }\end{array}$ & $k \lambda[\%]$ \\
\hline 3366 Marcellus & & 44.7 & \multirow{6}{*}{48.9} & \multirow{13}{*}{5.8} & 19.2 & \multirow{6}{*}{19.3} & \multirow{13}{*}{5.1} & 31.9 & \multirow{6}{*}{36.1} & \multirow{13}{*}{6.0} \\
\hline 3367 Marcellus & $\frac{1}{2}$ & 53.5 & & & 20.2 & & & 40.0 & & \\
\hline 3368 Marcellus & $\frac{.3}{0}$ & 50.7 & & & 19.8 & & & 37.5 & & \\
\hline 3369 Marcellus & 苋 & 47.4 & & & 19.4 & & & 34.4 & & \\
\hline 3370 Marcellus & $\frac{2}{2}$ & 49.4 & & & 18.4 & & & 37.1 & & \\
\hline 3371 Marcellus & & 47.9 & & & 18.5 & & & 35.6 & & \\
\hline & & & & & & & & & & \\
\hline 3372 Marcellus & \multirow{6}{*}{$\frac{\overline{0}}{\frac{\bar{c}}{\frac{\pi}{0}}}$} & 56.4 & \multirow{6}{*}{51.8} & & 22.4 & \multirow{6}{*}{20.25} & & 41.4 & \multirow{6}{*}{38.3} & \\
\hline 3373 Marcellus & & 52.7 & & & 22.1 & & & 38.0 & & \\
\hline 3374 Marcellus & & 58.8 & & & 21.9 & & & 44.2 & & \\
\hline 3375 Marcellus & & 54.5 & & & 18.8 & & & 42.0 & & \\
\hline 3376 Marcellus & & 45.9 & & & 18.0 & & & 33.9 & & \\
\hline 3377 Marcellus & & 42.7 & & & 18.3 & & & 30.5 & & \\
\hline
\end{tabular}

Table 12 Ultrasonic properties of Marcellus rock. ${ }^{* 1}$ from seismic P-wave velocity, depending on the location; ${ }^{* 2}$ from seismic; ${ }^{* 3}$ stress-strain tests; ${ }^{* 4}$ average from all measured samples.

\begin{tabular}{|l|c|c|c|c|}
\hline \multicolumn{1}{|c|}{ Parameter } & $\begin{array}{c}\text { Inks et al., } \\
2015^{* 1}\end{array}$ & $\begin{array}{c}\text { Gaiser et al., } \\
2011^{* 2}\end{array}$ & $\begin{array}{c}\text { Lora et al., } \\
2016^{* 3}\end{array}$ & This study \\
\hline P-wave anisotropy & $4.15-8.55 \%$ & $5 \%$ & - & $3 \%$ \\
\hline S-wave anisotropy & - & $3-5 \%$ & - & $3 \%$ \\
\hline Young's Modulus & - & - & $17 \mathrm{GPa}$ & $52.4 \mathrm{GPa}^{* 4}$ \\
\hline Poisson's Ratio & - & - & 0.13 & $0.33^{* 4}$ \\
\hline
\end{tabular}

Tables $9-11$ present results of ultrasonic measurements on Marcellus rock.

P-wave velocities in Marcellus samples do not show any significant changes depending on the direction of the cut out. The Average P-wave velocity of the samples cut out perpendicularly is about $200 \mathrm{~m} / \mathrm{s}$ lower than the velocity of samples cut out parallel, which gives $k$ coefficient equaled $3.2 \%$. $k$ for $\mathrm{S}$-wave is even lower. Young's modulus and Poisson's ratio of measured samples are higher than moduli available in the literature $(E-17 \mathrm{GPa}, v-$ 0.13 , in single stage triaxial compressional tests, Lora et al., 2016). Measured moduli are more similar to moduli of pure calcite which Domenico (1984) described. Table 12 shows anisotropy factors and elastic moduli of Marcellus rock available in literature. It should be mentioned, that Table 12 presents comparison of anisotropy factors calculated in various conditions and using various methodologies, thus these data should be rather an approximate reference point. Nevertheless, paying attention to the data (e.g. Lora et al., 2016; McGinley, 2015), it can be stated, that Marcellus samples of this study should not be considered as typical Marcellus shale rock. It can be assumed, that tested samples come from interbedded limestone layers in Marcellus shale, as suggests Harper et al. (2004).

Table 13 and Figure 2 show the brittleness index based on ultrasonic measurements.

The Samples of Mancos shale have low Young's moduli and relatively high Poison's ratios due to the high content of clay minerals. It causes that brittleness
Table 13 Brittleness indexes of Mancos and Marcellus samples. BRIT Grieser and Bray, 2007 brittleness index of single sample, $B R I T_{\text {average }}$ - average brittleness index of samples cut out in the same direction.

\begin{tabular}{|c|c|c|c|}
\hline \multicolumn{2}{|l|}{ Sample ID } & $\begin{array}{c}\text { BRIT Griesier \& Bray, } 2007 \\
{[\%]}\end{array}$ & $\begin{array}{c}B R I T_{\text {average }} \\
{[\%]}\end{array}$ \\
\hline 3268 Mancos & \multirow{6}{*}{ 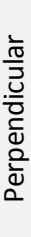 } & \multicolumn{2}{|l|}{-} \\
\hline 3269 Mancos & & 31.55 & \multirow{5}{*}{29.61} \\
\hline 3270 Mancos & & 25.75 & \\
\hline 3271 Mancos & & 29.75 & \\
\hline 3272 Mancos & & 30.20 & \\
\hline 3273 Mancos & & 30.80 & \\
\hline 3274 Mancos & \multirow{6}{*}{$\frac{\overline{0}}{\overline{\frac{\pi}{\sigma}}}$} & 25.80 & \multirow{6}{*}{25.95} \\
\hline 3275 Mancos & & 25.75 & \\
\hline 3276 Mancos & & 25.20 & \\
\hline 3277 Mancos & & 26.20 & \\
\hline 3278 Mancos & & 27.25 & \\
\hline 3279 Mancos & & 25.50 & \\
\hline 3366 Marcellus & \multirow{6}{*}{ 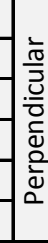 } & 44.20 & \multirow{6}{*}{43.03} \\
\hline 3367 Marcellus & & 43.95 & \\
\hline 3368 Marcellus & & 43.25 & \\
\hline 3369 Marcellus & & 43.60 & \\
\hline 3370 Marcellus & & 41.50 & \\
\hline 3371 Marcellus & & 41.65 & \\
\hline 3372 Marcellus & \multirow{6}{*}{$\frac{\overline{0}}{\frac{\pi}{\frac{\pi}{\pi}}}$} & 47.70 & \multirow{6}{*}{44.19} \\
\hline 3373 Marcellus & & 47.10 & \\
\hline 3374 Marcellus & & 46.15 & \\
\hline 3375 Marcellus & & 40.25 & \\
\hline 3376 Marcellus & & 40.90 & \\
\hline 3377 Marcellus & & 43.05 & \\
\hline
\end{tabular}




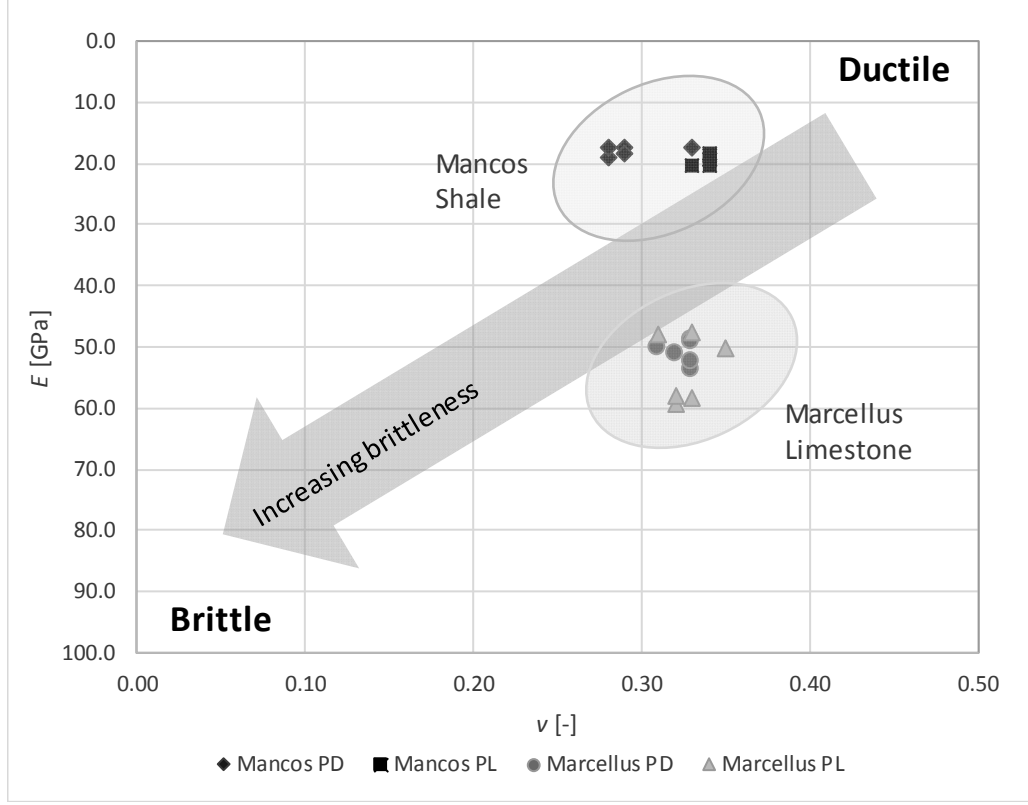

Fig. 2 Brittleness index BRIT Grieser and Bray, 2007 of Mancos and Marcellus samples.

Table 14 Brittleness index of measured samples (XRD data from Table 3). 0.0 - purely ductile rock, 1.0 - purely brittle rock.

\begin{tabular}{|l|c|c|c|}
\hline \multicolumn{1}{|c|}{ Sample ID } & BRIT $_{\text {Jarvie et al., 200 }}$ & BRIT $_{\text {Wang \& Gale, 2009, modified }}$ & BRIT $_{\text {Jin et al., 2014a, 2014b }}$ \\
\hline 3271 Mancos & 0.44 & 0.54 & 0.76 \\
\hline 3274 Mancos & 0.46 & 0.55 & 0.84 \\
\hline 3366 Marcellus & 0.08 & 0.09 & 0.92 \\
\hline 3372 Marcellus & 0.02 & 0.02 & 0.92 \\
\hline
\end{tabular}

index of all samples is low. The average brittleness index is higher for samples cut out perpendicularly what is determined by lower Poisson's ratios. Values

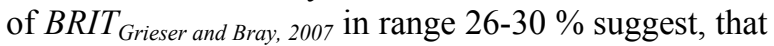
during the fracturing a basic two-wings fracture would be create (Rickman et. al, 2008; Kasza, 2013). Fracture network in the formation would be not extended, thus recommended treatment fluid should be crosslinked gel of high viscosity or energized foam with high content of shale minerals stabilizer. Proppant concentration should be high because of a high transport capability of the fluid, while fluid volume and delivery rate should be low due to the volume of fractures and high viscosity of fluid. In formations of low Young's modulus may appear an intensification of the embedment phenomena (Alramahi and Sundberg, 2012). The grains of the proppant could be pressed into the surface of the fracture, so as the effective width of the fracture would be decreased and therefore fracture conductivity may decrease. In the light of above it can be stated that tested samples of Mancos shale would be hard to fracturing.In the case of the measured
Marcellus rocks, $E$ to $v$ relation-based brittleness indexes are higher than in Mancos shale. It is caused by significantly higher Young's moduli, which is more similar to Young's modulus of pure calcite (Domenico, 1984). These samples do not show differences in brittleness indexes depending on the direction of the cut out, which is caused by low anisotropy.

Additionally for a sake of comparison, authors calculated the brittleness index using three different methods. Table 14 presents brittleness indexes of the measured samples.

Table 14 shows large differences in brittleness indexes depending on used equation. In Jarvie's et al. (2007) and Wang's and Gale's (2009) equations brittle mineral is quartz or quartz and dolomite respectively. In the other hand in Jin's et al. (2014a, 2014b) equation brittle minerals are feldspar, mica and calcite as well. Especially in the case of measured Marcellus samples difference in assumptions during calculations of brittleness indexes is well visible. Testing of the same sample depending on the different approaches may give different results, therefore 
brittleness indexes (mineral and geomechanical) should be used complementary.

It should be mentioned as well, that the extrapolation of laboratory tests to the field encounters some problems. Core sample preparation, influence of temperature, changes of inclusion fluids cause the damage of micro-cracking in the sample. Thus core tests usually cannot represent field conditions and are a simplification of field environments (Zhang et al., 2016). However, laboratory core measurements still find application as a supplement to well logging data during designing of hydraulic fracturing treatment.

\section{CONCLUSIONS}

Laboratory ultrasonic measurements provide useful geomechanical data allowing calculate anisotropy and brittleness indexes of the rock. These kinds of measurements may be used as supplement or calibration of the geophysical results. This paper shows, that anisotropy of the rock has a noticeable influence on the elastic moduli-based brittleness index calculations. However, a lot of various definitions of brittleness indexes are used in oil and gas industry (geomechanical, mineral-based and other) and each of them gives different results. Therefore brittleness indexes should be used complementary to better describe fracability of the rock.

\section{ACKNOWLEDGEMENTS}

This paper is based on the results from statutory work. Archive no. DK-4100-55/17.

\section{REFERENCES}

Alramahi, B. and Sundberg, M.I.: 2012, Proppant embedment and conductivity of hydraulic fractures in shales. ARMA, 12-291, 1-6.

Andreev, G.: 1995, Brittle faliure of rock materials, CRC Press.

Bała, M.: 1990, Sedimentary rock classification based on their modulus of elastiity estimated from wave images. Przegląd Geologiczny, 38, 12, 556-559, (in Polish with English summary).

Bourbie, T., Coussy, O. and Zinszner, B.: 1987, Acoustic of porous media. Editions Technip, 175-242.

Britt, L. and Schoeffler, J.: 2009, The geomechanics of a shale play: what makes a shale perspective! SPE 125525, 1-9. DOI: 10.2118/125525-MS

Chandler, M., Meredith, P., Brantut, N. and Crawford, B.: 2016, Fracture toughness anisotropy in shale. J. Geophys. Res., 121, 3, 1706-1729. DOI: $10.1002 / 2015 J B 012756$

Domenico, S.: 1984, Rock lithology and porosity determination from shear and compressional wave velocity. Geophysics, 49, 8, 1188-1195. DOI: $10.1190 / 1.1441748$

Fjaer, E., Holt, R., Horsrud, P., Raanen, A. and Risnes, R.: 2008, Petroleum related rock mechanics, second edition. Elsevier.

Gaiser, J., Chaveste, A., Edrich, M., Rebec, T. and Verm, R.: 2011, Seismic traveltime anisotropy of the Marcellus Shale: feasibility study for fracture characterization. Recovery, 1-4.

DOI: $10.3997 / 2214-4609.20146787$

Grieser, B. and Bray, J.: 2007, Identification of production potential in uncoventional reservoirs. SPE 106623, 16. DOI: $10.2118 / 106623-\mathrm{MS}$

Gong, Q. and Zhao, J.: 2007, Influence of rock brittleness on TMB penetration rate in Singapore granite. Tunelling and Underground Space Technology, 22, 3, 317-324. DOI: 10.1016/j.tust.2006.07.004

Goodway, B., Perez, M., Varsek, J. and Abaco, C.: 2010, Seismic petrophysics and isotropic-anisotropic AVO methods for unconventional gas exploration. The Leading Edge, 29, 12, 1500-1508. DOI: $10.1190 / 1.3525367$

Harper, J., Laughrey, C., Kostelnik, J., Gold, D. and Doden, A: 2004, Trenton and Black River carbonates in the Union Furnace area of Blair and Huntingdon Countries, Pennsylvania. Field Trip Guidebook for the Eastern Section AAPG Annual Meeting. Pensylvania Geological Survey, 2004.

Holt, R., Fjaer, E., Nes, O. and Alassi, H.: 2011, A shaly look at brittleness. ARMA 11-366.

Inks, T., Engelder, T., Jenner, E., Golob, B., Hocum, J. and O'Brien, D.: 2015, Marcellus fracture characterization using P-wave azimuthal velocity attributes: comparison with production and outcrop data. Interpretation, 3, 3, SU1-SU15. DOI: $10.1190 /$ INT-2014-0215.1

Jarvie, D., Hill, R., Ruble, T. and Pollastro, R.: 2007, Unconventional shale-gas systems: The Mississippian Barnett Shale of north-central Texas as one model for thermogenic shale-gas assessment. AAPG Bulletin, 91, 4, 475-499. DOI: 10.1306/12190606068

Jin, X., Shah, S., Roegiers, J-C. and Zhang, B: 2014a, Fracability evaluation in shale reservoirs - an integrated petrophysics and geomechanics approach. SPE 168589, SPE Conference Paper, 1-14. DOI: $10.2118 / 168589-M S$

Jin, X., Shah, S., Truax, J. and Roegiers, J-C.: 2014b, A practical petrophysical approach for brittleness prediction from porosity and sonic logging in shale reservoirs. SPE 170972, SPE Conference Paper, 1-21. DOI: $10.2118 / 170972-M S$

Kasza, P.: 2013, Effective fracturing of shales in Poland. Nafta-Gaz, 11, 807-813, (in Polish with English abstract).

Kowalska, S.: 2013, Quantitative analysis of the mineral composition of rock containing clay minerals by Rietveld method. Nafta-Gaz, 12, 894-902, (in Polish with English abstract).

Luan, X., Di, J., Wei, X., Li, K., Qian, J. and Xie, P.: 2014, Laboratory measurements of brittleness anisotropy in synthetic shale with different cementation. Society of Exploration Geophysicist, Annual Meeting, Denver 2014, 3005-3009. DOI: 10.1190/segam2014-0432.1

Lora, R., Ghazanfari, E. and Izquiredo, E.: 2016, Geomechanical characterization of Marcellus shale. Rock Mech. and Rock Eng., 49, 9, 3403-3424. DOI 10.1007/s00603-016-0955-7

McGinley, M.: 2015, The effects of fracture orientation and anisotropy on hydraulic fracture conductivity in the Marcellus shale. A Thesis. Texas A\&M University, 37 pp. 
Mokhtari, M.: 2015, Characterization, of anisotropy in organic-rich shales: shear and tensile failure, wave velocity, matrix and fracture permeability. A Thesis. Colorado School of Mines, $13 \mathrm{pp}$.

Moska, R.: 2017, Laboratory acoustic measurements for the needs of hydraulic fracturing. Nafta-Gaz, 2, 81-89, (in Polish with English abstract).

DOI: 10.18668/NG.2017.02.02

Perez, R. and Marfurt, K.: 2014, Mineralogy-based brittleness prediction from surface seismic data: application to the Barnett Shale. Interpretation, 2, 4, T255-T271. DOI: 10.1190/INT-2013-0161.1

Rickman, R., Mullen, M., Petre, E., Greiser, B. and Kundert, D.: 2008 , A practical use of shale petrophysics for stimulation designing optimalization: all shale plays are not clones of the Barnett Shale. SPE 115258, 111. DOI: $10.2118 / 115258-\mathrm{MS}$

Sarker, R. and Batzle, M.: 2010, Anisotropic elastic moduli of the Mancos B shale - an experimental study. SEG 2010-2600, 2600-2605. DOI: 10.1190/1.3513381

Sone, H. and Zoback, M.: 2013, Mechanical properties of the shale-gas reservoir rocks - part 1: static and dynamic elastic properties and anisotropy. Geophysics, 78, 5, D381-D392. DOI: $10.1190 /$ geo2013-0050.1

Stan-Kłeczek, I.: 2016, The study of the elastic properties of carbonate rocks on a base of laboratory and field measurements. Acta Mont. Slovaca. 21, 1, 76-83. DOI: 10.3390/ams21010075

Sun, S., Wang, P., Yang, X., Li, J., Sun, B. and Liu, K.: 2013, Integrated prediction of shale oil reservoir using pre-stack algorithms for brittleness and fracture detection. International Petroleum Technology Conference, IPTC 17048.

DOI: $10.2523 /$ IPTC-17048-MS
Środoń, J., Drits, V., McCarty, D., Hsieh, J. and Eberl, D.: 2001, Quantitative X-ray diffraction analysis of claybearing rocks from random preparations. Clays and Clay Miner., 49, 6, 514-528. DOI: $10.1346 / C C M N .2001 .0490604$

Tarasov, B. and Potvin, Y.: 2013, Universal criteria for rock brittleness estimation under triaxial compression. Int. J. Rock Mech. Min. Sci., 59, 57-69. DOI: 10.1016/j.ijrmms.2012.12.011

Thomsen, L.: 1986, Weak elastic anisotropy. Geophysics, 51, 10, 1954-1966. DOI: 10.1190/1.1442051

Wang, F. and Gale, J.: 2009, Screening criteria for shale-gas systems. Gulf Coast Association of Geological Societes Transactions, 59, 779-793.

Wanniarachichi, A., Ranjith, P., Perera, S., Lashin, A., Al Arifi, N. and Li, J.: 2015, Current options on foambased hydro-fracturing in deep geological reservoirs. Geomech. Geophys. Geo-energ. Geo-resour., 1, 3-4, 121-134. DOI 10.1007/s40948-015-0015-x

Winkler, K. and Murphy, W.: 1995, Acoustic Velocity and Attenuation in Porous Rocks. Rock Physics and Phase Relations: A Handbook of Physical Constants, 21-34.

Zhang, D., Ranjith, P.G. and Perera, M.S.A.: 2016, The brittleness indices used in rock mechanic and their applications in shale hydraulic fracturing: a review. J. Petrol. Sci. Eng., 143, 158-170. DOI: 10.1016/j.petrol.2016.02.011

Živor, R., Vilhelm, J., Rudajev, V. and Lokajíček, T.: 2011, Measurement of P- and S-wave velocities in a rock massif and its use in estimating elastic moduli. Acta Geodyn. Geomater., 8, 2 (162), 157-167. 